\title{
Projeto, adaptação, instalação e testes preliminares para um sistema de medida de energia de fratura de materiais cerâmicos pelo método da cunha
}

\section{(Design, adaptation, installation and preliminary testing of a fracture energy measuring system for ceramic materials based on the wedge splitting method)}

\author{
S. Ribeiro', C.C.D. Exposito ${ }^{2}$, J.A. Rodrigues ${ }^{2}$ \\ ${ }^{\prime}$ Departamento de Engenharia de Materiais - DEMAR, Escola de Engenharia de Lorena - EEL, \\ Universidade de S. Paulo - USP, Lorena, SP 12600-970 \\ ${ }^{2}$ Grupo de Engenharia de Microestrutura de Materiais - GEMM, Departamento de Engenharia de Materiais \\ DEMa Universidade Federal de S. Carlos - UFSCar, S. Carlos, SP 13565-905 \\ sebastiao_eel@yahoo.com.br
}

\begin{abstract}
Resumo
Este trabalho discute o projeto e a instalação dos dispositivos para determinação da energia de fratura em materiais cerâmicos utilizando o método da cunha patenteado, além dos resultados preliminares de carga máxima e energia de fratura para duas argamassas. Para testar o sistema, e conseqüentemente implantar o método, foram preparadas argamassas com cimento Portland, areia e água, moldadas, curadas a $25{ }^{\circ} \mathrm{C}$ por 7 dias e secas a $50{ }^{\circ} \mathrm{C}$ por $48 \mathrm{~h}$. Foram caracterizadas duas proporções de areia:cimento de $3: 1$ e $2: 1$. A partir dos testes preliminares foram estabelecidas as condições de propagação estável da trinca. A trinca percorre um plano imaginário definido por ranhuras laterais da amostra. O método está plenamente instalado e pronto para o desenvolvimento de trabalhos sistemáticos correlacionando energia de fratura e microestruturas de refratários e cerâmicas em geral, incluindo materiais para construção civil. Além do pleno funcionamento do método para temperatura ambiente, os resultados mostram que a técnica é sensível aos aspectos microestruturais do material caracterizado, distinguindo, neste trabalho, entre as duas composições de argamassas empregadas.

Palavras-chave: método da cunha, propagação estável de trinca, energia de fratura, argamassa, cimento Portland.
\end{abstract}

\begin{abstract}
This paper discusses the design and installation of devices for determining fracture energy in ceramic materials based on the patented wedge splitting test. Preliminary results of the maximum load and fracture energy for two different mortar compositions are also shown. To test the system and apply the method, mortars made of Portland cement, sand and water were molded and cured at $25^{\circ} \mathrm{C}$ for 7 days and dried at $50^{\circ} \mathrm{C}$ for $48 \mathrm{~h}$. Two cement-sand composition ratios were characterized: 3:1 and 2:1. Preliminary tests were applied to establish stable crack propagation conditions. The crack was found to propagate through an imaginary plane defined by lateral grooves in the sample. The system is fully installed and ready for the development of systematic works correlating fracture energy and microstructure of refractory and ceramic materials in general, including materials for civil construction. In addition to demonstrating the efficiency of the method at room temperature, the results revealed that the technique is sensitive to microstructural aspects of the characterized material, since it successfully distinguished between the two mortars employed in this work.

Keywords: wedge splitting method, stable crack propagation, fracture energy, mortar, Portland cement.
\end{abstract}

\section{INTRODUÇÃO}

Refratários são materiais com microestrutura complexa e grosseira, resistentes a altas temperaturas e usados predominantemente como revestimentos de fornos e equipamentos industriais para processamento de materiais em elevadas temperaturas. Sua microestrutura é projetada para minimizar danos por choque térmico, já que esses materiais são usualmente submetidos a abruptas mudanças de temperatura.
De um modo geral, sua microestrutura é constituída por uma parte mais fina, podendo ter tamanhos de alguns nanômetros, chamada de matriz e outra mais grossa formada por agregados com tamanhos de até alguns milímetros [2].

Algumas propriedades dos refratários requerem maior atenção, entre elas destacam-se o módulo de ruptura $\left(\sigma_{\mathrm{f}}\right)$, o coeficiente de expansão térmica linear $(\alpha)$, o módulo de elasticidade (E), a condutividade térmica $(\mathrm{k})$ e a resistência ao dano por choque térmico [2]. 
Para avaliação da resistência ao dano por choque térmico, Hasselman [3-12] escreveu duas importantes equações:

$$
\begin{aligned}
& R^{\prime \prime \prime}=\frac{\gamma_{w o f} E}{\sigma_{f}^{2}(1-v)} \\
& R_{s t}=\left[\frac{\gamma_{w o f}\left(1-v^{2}\right)}{E \alpha^{2}}\right]^{\frac{1}{2}}
\end{aligned}
$$

em que R'" é o parâmetro de resistência ao dano por choque térmico, $\mathrm{R}_{\mathrm{st}}$ é o parâmetro de estabilidade da trinca sob tensão térmica, $\gamma_{\text {wof }}$ é a energia de fratura e $v$ é a razão de Poisson.

Comose vê, aenergia de fraturaéum termoimportantíssimo na previsão da resistência ao dano por choque térmico dos refratários, cujos resultados servem para desenhar novos materiais e aperfeiçoar aqueles já comercialmente disponíveis no mercado.

A energia de fratura pode ser definida como o trabalho médio por unidade de área projetada de fratura para propagar uma trinca, representada pela soma de distintas energias consumidas durante o processo de propagação e pode ser determinada a partir da curva de carga-deslocamento e da seguinte equação [4, 8-10, 13-21]:

$$
\gamma_{\text {wof }}=\frac{1}{2 \mathrm{~A}} \int \mathrm{F}_{\mathrm{M}} \mathrm{d} \delta
$$

em que A é a área projetada da superfície de fratura, $\mathrm{F}_{\mathrm{M}}$ é a carga vertical aplicada pela máquina de ensaio e $\delta$ é o deslocamento do atuador da máquina. $\mathrm{O}$ valor da integral $\int \mathrm{F}_{\mathrm{M}} \mathrm{d} \delta$ é determinado pela área sob a correspondente curva carga-deslocamento.

Para se avaliar a energia de fratura, a condição de propagação estável da trinca deve ser rigorosamente obedecida e para isso, a energia acumulada na amostra e na máquina de ensaios durante a deformação elástica deve ser a menor possível e transformada em energia de superfície de modo controlado. A condição necessária para tal pode ser descrita pela seguinte inequação:

$$
-\frac{\mathrm{d}\left(\mathrm{U}_{\mathrm{M}}+\mathrm{U}_{\mathrm{S}}\right)}{\mathrm{dc}} \leq \frac{\mathrm{dW}_{\mathrm{fs}}}{\mathrm{dc}}
$$

em que $\mathrm{d}\left(\mathrm{U}_{\mathrm{M}}+\mathrm{U}_{\mathrm{S}}\right) / \mathrm{dc}$ é a variação das energias elásticas armazenadas na máquina $\left(\mathrm{U}_{\mathrm{M}}\right)$ e na amostra $\left(\mathrm{U}_{\mathrm{S}}\right)$ por unidade de propagação de trinca $(\mathrm{dc})$, sendo essa, portanto, a variação da energia potencial, e $\mathrm{dW}_{\mathrm{fs}} / \mathrm{dc}$ é a variação da energia de superfície $\left(\mathrm{dW}_{\mathrm{fs}}\right)$ por unidade de propagação de trinca [11, 16, 18], representando a variação da energia consumida durante o processo de propagação.

Até pouco tempo atrás a energia de fratura de refratários era predominantemente determinada pelo método da barra prismática entalhada e flexionada em três pontos, conforme esquematizado na Fig. 1 [4, 9, 13, 17, 18]. Normalmente, a barra possui medidas de altura, h, e espessura, b, de $25 \mathrm{~mm}$ x $25 \mathrm{~mm}$ ou $40 \mathrm{~mm}$ x $40 \mathrm{~mm}$, com entalhe, c, em torno de
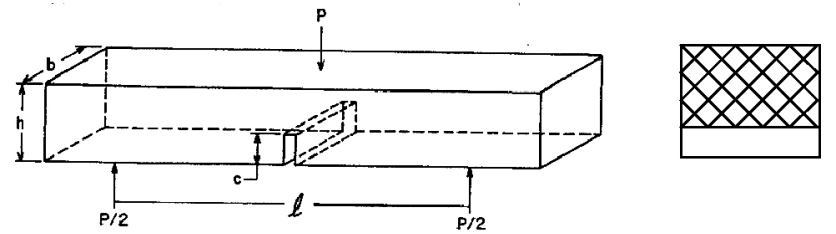

(a)

(b)

Figura 1: (a) Geometria e dimensões da amostra para medida da energia de fratura pelo método da flexão em três pontos [18] e (b) seção transversal, mostrando a região por onde a trinca se propaga.

[Figure 1: (a) Geometry and dimensions of test specimens used to measure the fracture energy by three-point bending test [18] and (b) cross section, showing the region where the crack propagates.]


Figura 2: (a) Representação esquemática do método da cunha e detalhes da construção das peças de transmissão de carga. $F_{H}$ e $\beta$ são, respectivamente: força horizontal para a abertura da trinca e ângulo da cunha [10], (b) geometria da amostra, dimensões e secção transversal mostrando a região por onde a trinca propaga (medidas em mm) [11], (c) detalhes do entalhe, das ranhuras laterais e da secção de fratura após teste.

[Figure 2: (a) Schematic representation of the wedge splitting method and details for the construction of the load transmission pieces. $F_{H}$ and $\beta$ are horizontal load and wedge angle, respectively [10], (b) geometry of the specimen, dimensions and cross section showing the region where the crack propagates (dimensions in $\mathrm{mm}$ ) [11], (c) details of rabbet, lateral grooves and fracture section after test .] 
0,4 da altura, restando assim pequena área de fratura. Dessa forma, a área do ligamento íntegro restante pode não ser mais representativa da microestrutura do material, principalmente se existirem agregados com tamanhos grandes comparados com essas dimensões. Por isso, a caracterização mecânica da fratura, de forma mais rigorosa, requer um método que permita propagação estável de trinca para dimensões de amostras suficientemente grandes, principalmente nas suas dimensões através das quais a trinca se propaga. Amostras com maiores dimensões são compatíveis com a característica grosseira da microestrutura dos refratários.

Interessado em determinar parâmetros da mecânica da fratura, em 1986 foi patenteado um método chamado "wedge splitting test", que é o método da abertura da trinca empregando-se uma cunha, ou simplesmente "Método da cunha" [1]. Como pode ser visto na Fig. 2a, a cunha transmite carga para dois roletes laterais e estes para os encostos que produzem as forças horizontais, $\mathrm{F}_{\mathrm{H}}$, na amostra sob teste. A Fig. $2 \mathrm{~b}$ apresenta a geometria da amostra e suas dimensões típicas [10, 11]. Na Fig. 2c vêem-se os detalhes do entalhe, das ranhuras laterais e da secção de fratura após teste, em corte que passa justamente pelo entalhe e ranhuras laterais.

As principais vantagens do método da cunha estão baseadas na possibilidade de se trabalhar com amostras grandes, para as quais o tamanho do agregado torna-se suficientemente pequeno para resultar em uma relação (agregado/dimensões transversais) pequena. Além disso, a força aplicada pela máquina de ensaio que é bastante reduzida devido ao efeito da cunha, diminuindo significativamente a energia elástica armazenada na máquina, facilitado a propagação estável da trinca durante o teste. A correlação entre a força aplicada pela máquina de ensaios, $\mathrm{F}_{\mathrm{M}}$, e a transmitida para a amostra, $\mathrm{F}_{\mathrm{H}}$, mostrando a influência do ângulo da cunha na relação carga aplicada e carga transmitida, pode ser avaliada observando a seguinte equação $[10,11,16,20]$ :

$$
\mathrm{F}_{\mathrm{H}}=\mathrm{F}_{\mathrm{M}} / 2 \cdot \operatorname{tg}(\beta / 2)
$$

O principal objetivo deste trabalho é mostrar as características, os dispositivos necessários e a implantação do método da cunha para a medida da energia de fratura visando melhorar a caracterização de concretos refratários e o projeto desses materiais. Para o estabelecimento dos procedimentos desse método, inicialmente foram usadas argamassas constituídas de cimento Portland e areia, que são baratos e de fácil preparação. Posteriormente, para validação, serão usados concretos refratários comerciais moldados.

\section{MATERIAIS E MÉTODOS}

Visando dar um caráter didático à descrição desse item, o mesmo foi dividido e apresentado em uma sequiência cronológica das atividades.
Projeto e construção do molde para a preparação das amostras de argamassas, que também será utilizado para moldagem de concretos refratários em uma próxima etapa do projeto da instalação do método da cunha

A Fig. 3 ilustra os detalhes e as medidas da amostra que serviu de base para o projeto, para a confecção do molde e
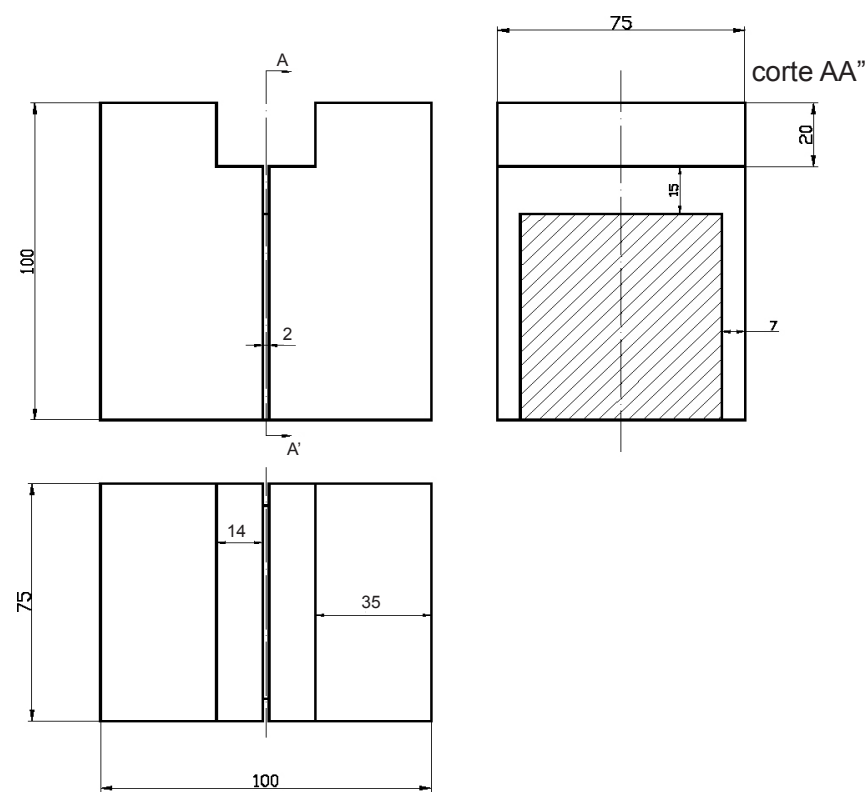

Figura 3: Desenho da amostra utilizada como base do projeto do molde, do dispositivo da cunha e dos acessórios de acoplamento à máquina de ensaio (dimensões em mm).

[Figure 3: Drawing of the sample used as reference for design of the mold, wedge device and accessories to couple to the test machine (dimensions in $\mathrm{mm}$ ).]

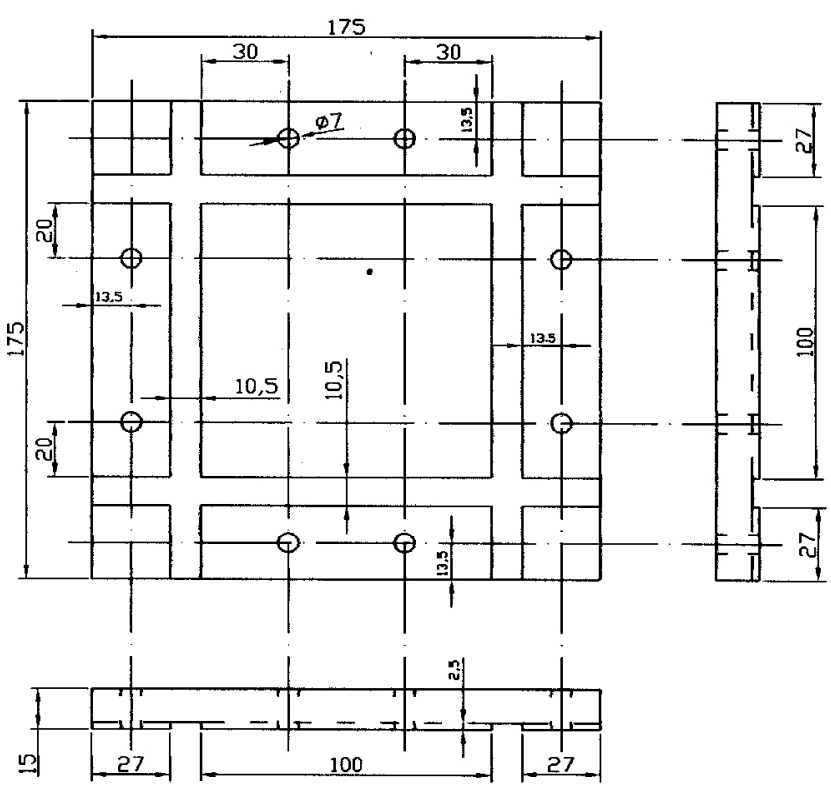

Figura 4: Desenho da lateral maior do molde de PVC (dimensões em $\mathrm{mm})$.

[Figure 4: Drawing of the bigger lateral part of the PVC mold (dimensions in $\mathrm{mm}$ ).] 


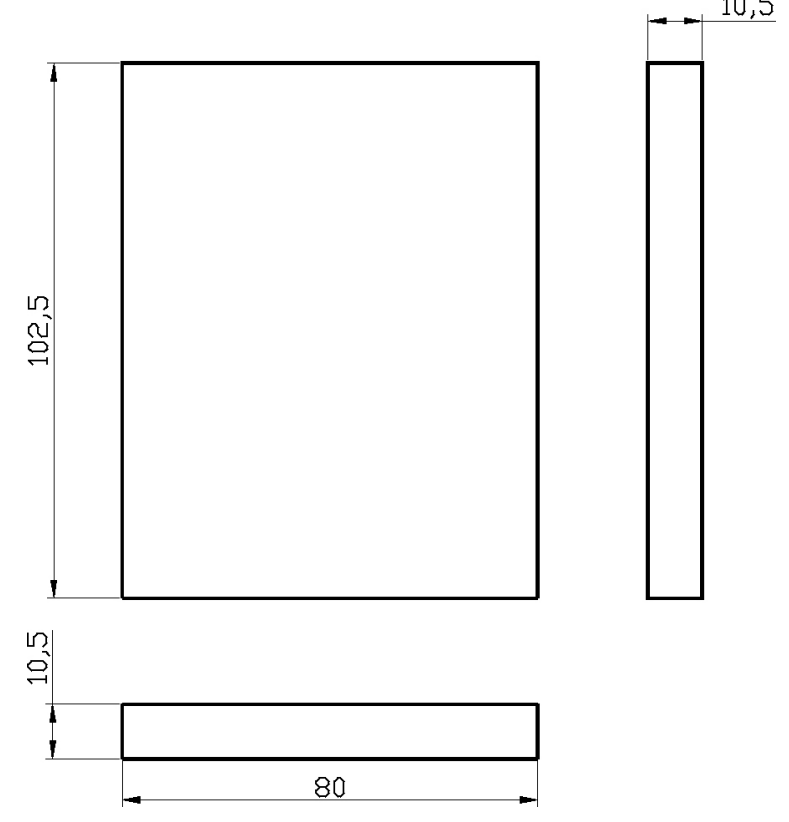

Figura 5: Desenho da lateral menor do molde de PVC (dimensões em $\mathrm{mm})$.

[Figure 5: Drawing of the smaller lateral part of the PVC mold (dimensions in $\mathrm{mm}$ ).]

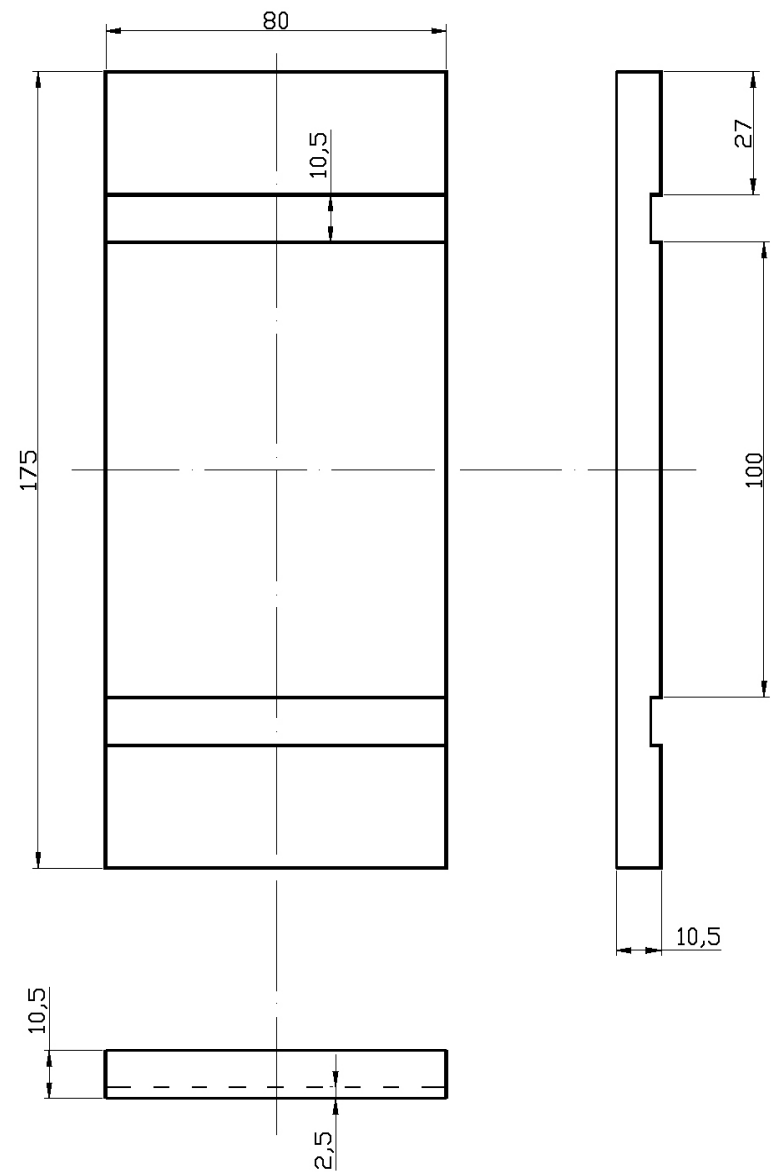

Figura 6: Desenho da base do molde de PVC (dimensões em $\mathrm{mm})$.

[Figure 6: Drawing of the base of the PVC mold (dimensions in mm).]

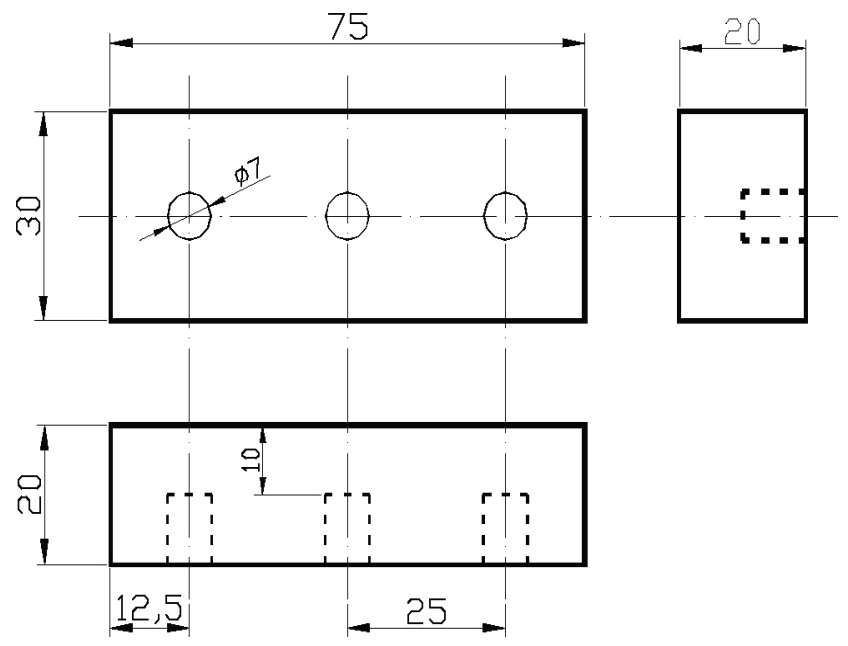

Figura 7: Desenho do macho de PVC (dimensões em mm).

[Figure 7: Drawing of the core of the PVC mold (dimensions in mm).]

do dispositivo da cunha. Essa escolha foi realizada a partir da avaliação da literatura referente ao método da cunha.

Nas Figs. 4 a 7 estão apresentadas às partes do molde de PVC, que foi projetado e construído para a elaboração das amostras com as dimensões constantes na Fig. 3. O conjunto constituído por essas peças é montado utilizandose parafusos de alumínio com $6,3 \mathrm{~mm}$ de diâmetro munidos de arruelas e porcas tipo borboleta.

As Figs. 4 e 5 mostram a parte lateral maior e menor do molde com suas respectivas medidas (em $\mathrm{mm}$ ).

A Fig. 6 mostra a base do molde, com suas respectivas medidas. Nessa base é montado o macho para produzir o rebaixo no corpo de prova onde é montado o dispositivo da cunha (encostos, roletes e cunha). O macho é mostrado na Fig. 7.

Projeto e construção do dispositivo que doravante será denominado "dispositivo da cunha".

As Figs. 8 a 10 mostram os desenhos das peças que constituem o dispositivo da cunha com suas respectivas medidas em $\mathrm{mm}$. Essas peças foram confeccionadas em aço VC131, temperadas, retificadas e polidas nas faces de contato metal-metal no momento do teste.
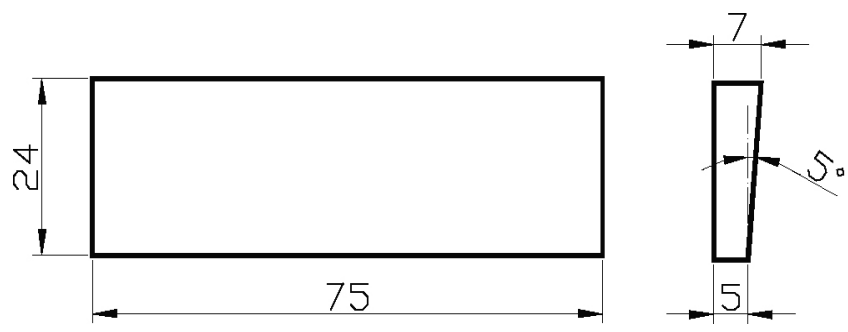

Figura 8: Desenho do encosto (dimensões em $\mathrm{mm}$ ). [Figure 8: Drawing of the support (dimensions in $\mathrm{mm}$ ).] 

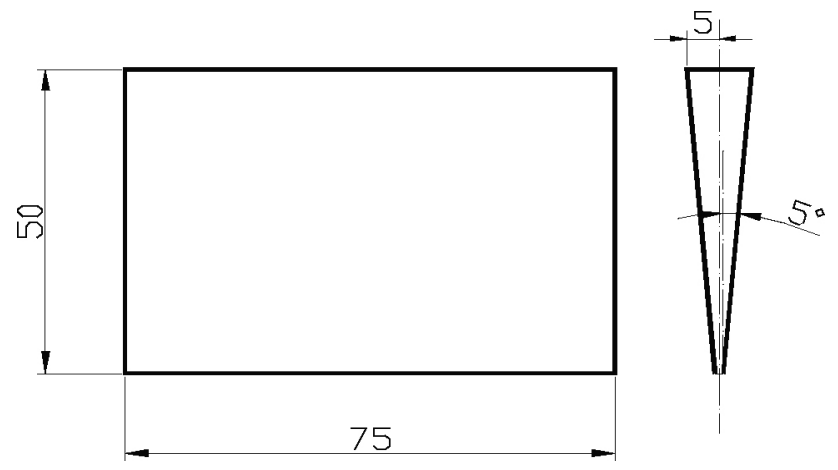

Figura 9: Desenho da cunha (dimensões em mm).

[Figure 9: Drawing of the wedge (dimensions in $\mathrm{mm}$ ).]

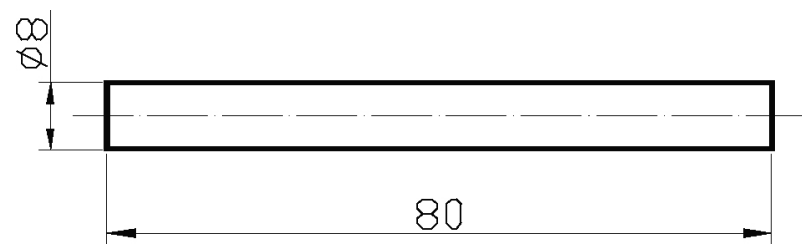

Figura 10: Desenho do rolete (dimensões em mm).

[Figure 10: Drawing of the roll (dimensions in $\mathrm{mm}$ ).]

Suporte para montagem dos roletes do dispositivo da cunha na amostra

A Fig. 11 mostra o desenho do dispositivo utilizado para montar os roletes e a cunha na amostra, servindo também para a fixação das facas de um extensômetro quando usado para o acompanhamento da abertura de trinca, se necessário. Este suporte foi confeccionado em alumínio com acabamento de usinagem, exceto na parte onde se montam as facas do extensômetro que são polidas.

Projeto das placas superior e inferior, que serão chamadas de acessórios de acoplamento à máquina de ensaio

As Figs. 12 e 13 mostram as duas placas cilíndricas desse acessório, feitas em aço VC131 temperado, sendo uma aparafusada na célula de carga da máquina de ensaio, chamada de placa superior, Fig. 12, e outra montada sobre o atuador da máquina, chamada de placa inferior, Fig. 13. A diferença básica dessas duas placas é que a placa inferior possui um apoio prismático de secção trapezoidal, que tem a finalidade de manter e apoiar a amostra ao longo de uma linha sob a sua base, fazendo com que a mesma não sofra resistência à abertura da trinca durante o ensaio.

Preparação das amostras para testar o método, os dispositivos e os acessórios mostrados nos itens anteriores

Para elaboração das amostras para os testes preliminares, foram usados: areia média lavada para construção civil, com tamanho médio de partículas de 500 um e tamanhos máximos e mínimos de $2800 \mu \mathrm{m}$ e $53 \mu \mathrm{m}$, respectivamente; cimento

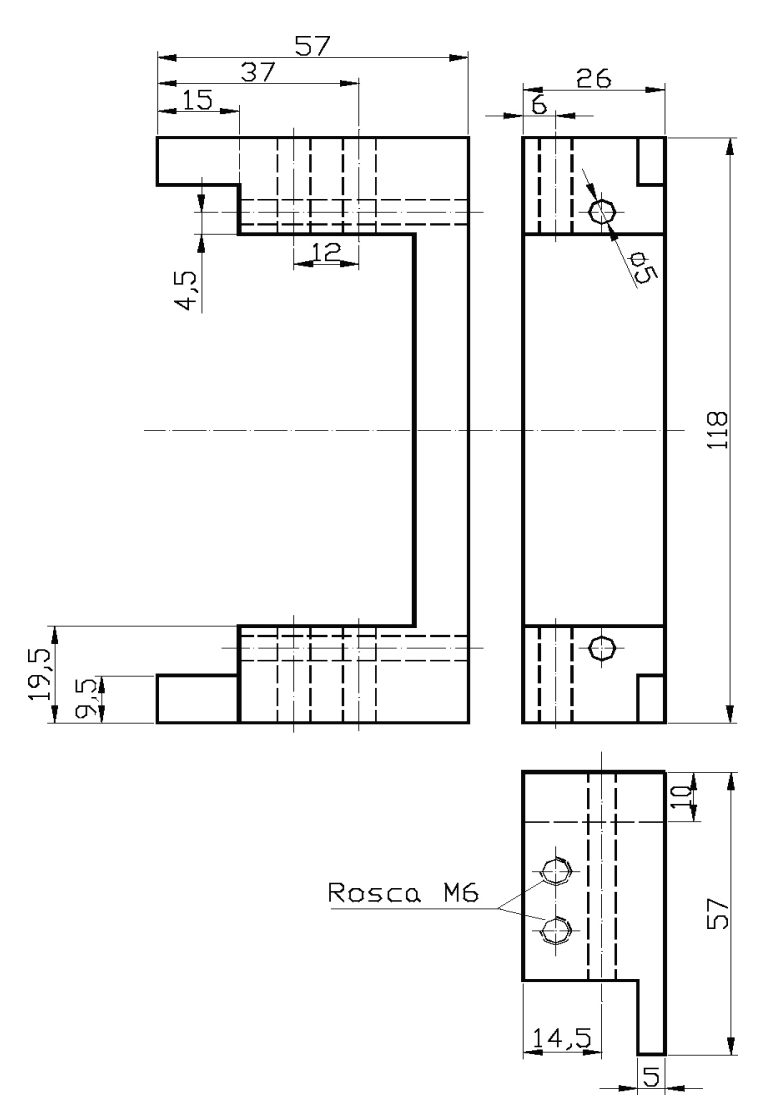

Figura 11: Desenho do dispositivo para montagem do sistema da cunha e de um extensômetro (dimensões em mm).

[Figure 11: Drawing of the device used to set up the wedge splitting system and one extensometer (dimensions in $\mathrm{mm}$ ).]
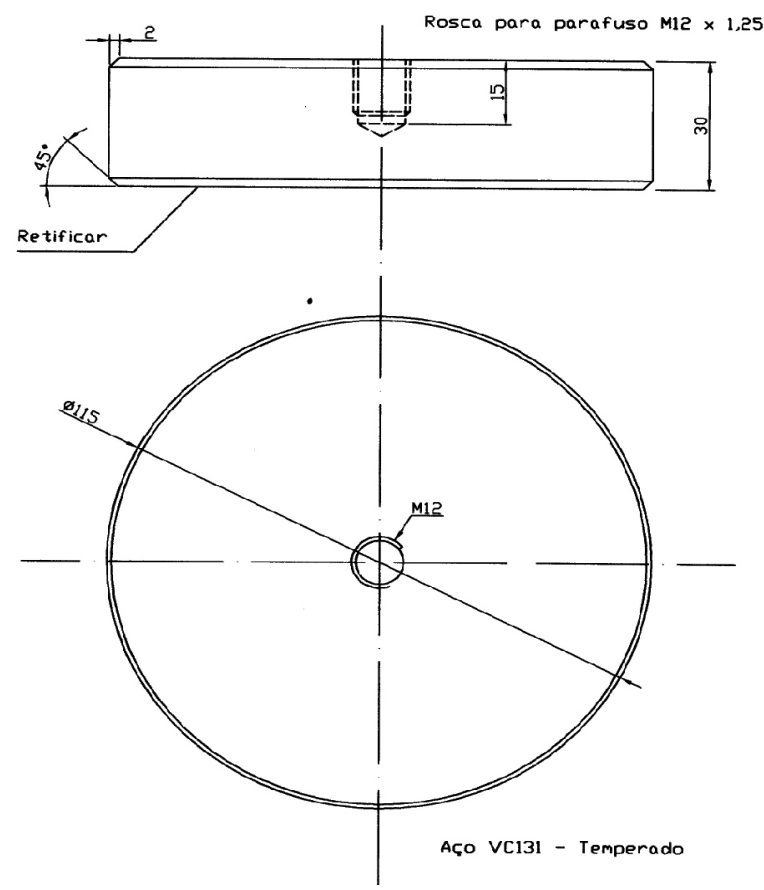

Figura 12: Desenho da placa superior do acessório de acoplamento à máquina de ensaio mecânico (dimensões em mm).

[Figure 12: Drawing of the upper plate to connect to the test machine (dimensions in $\mathrm{mm}$ ).] 


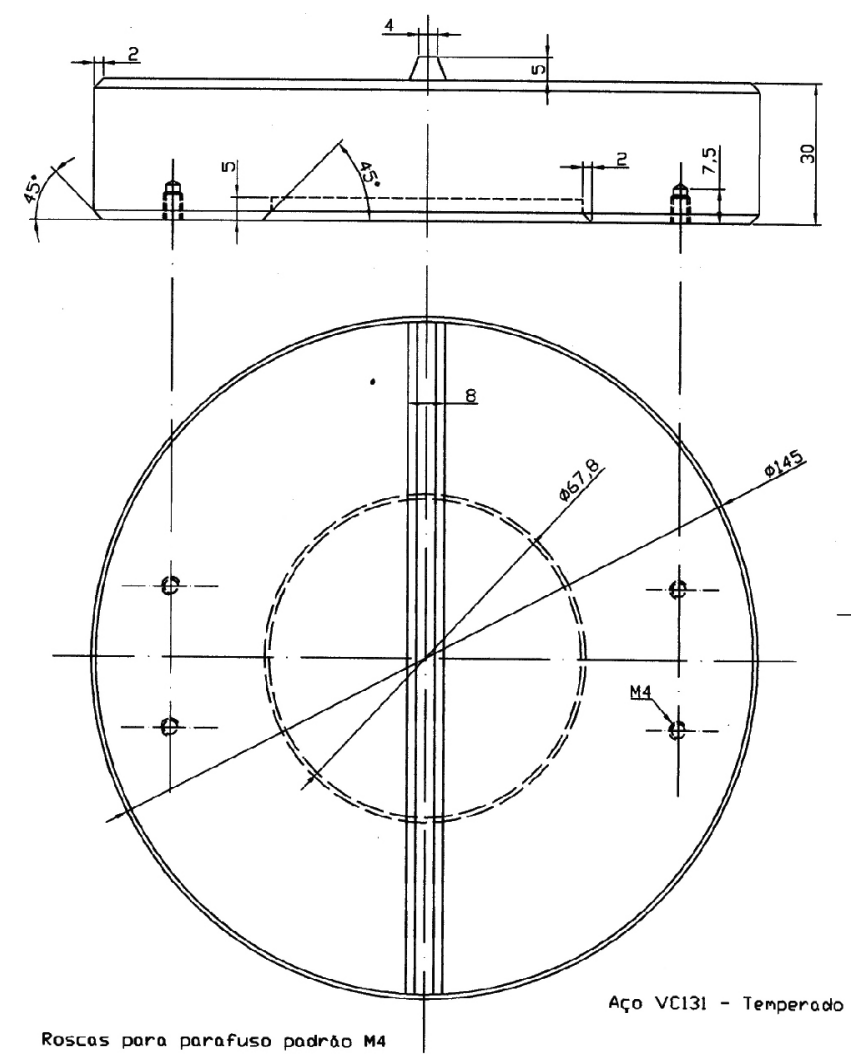

Figura 13: Desenho da placa inferior do acessório de acoplamento à máquina de ensaio (dimensões em $\mathrm{mm}$ ).

[Figure 13: Drawing of the lower plate to connect to the test machine (dimensions in $\mathrm{mm}$ ).]

Portland CPII-Z-32, Companhia de Cimento Portland Itaú, fabricado conforme Norma Brasileira NBR 11578/1991 e água potável.

Foram utilizadas duas composições mássicas em termos de areia:cimento de 2:1 e 3:1. A quantidade de água teve como base de cálculo 0,46 da massa de cimento. A areia e o cimento foram pesados e misturados previamente. Após mistura, a água foi adicionada aos poucos com o sistema de mistura em movimento, permitindo uma perfeita interação entre os três constituintes da argamassa.

A argamassa foi despejada no molde de $\mathrm{PVC}$ produzindo amostras nas dimensões de 100 x $100 \times 75 \mathrm{~mm}^{3}$. A moldagem foi realizada sobre uma mesa vibratória que auxilia a acomodação da argamassa no interior do molde e ajuda na expulsão de bolhas de ar. As amostras foram curadas por sete dias, em atmosfera saturada de água a $25{ }^{\circ} \mathrm{C}$ e secas a $50{ }^{\circ} \mathrm{C}$ por $48 \mathrm{~h}$.

Após secagem, as amostras foram retificadas nas suas bases com o objetivo de promover o alinhamento com o eixo da máquina de ensaio, ranhuras e entalhe. Esta condição é obrigatória para que as forças horizontais, $\mathrm{F}_{\mathrm{H}}$, estejam balanceadas entre os dois lados do entalhe. As ranhuras e o entalhe foram produzidos com disco diamantado com perfil quadrado e espessura de $1,5 \mathrm{~mm}$. O entalhe e as ranhuras tinham $2 \mathrm{~mm}$ de espessura. As suas profundidades eram de 15 e $7 \mathrm{~mm}$, respectivamente.
Testes propriamente ditos com as respectivas avaliações dos resultados

A Fig. 14 mostra um desenho técnico do arranjo experimental envolvendo uma amostra e o dispositivo da cunha. Este sistema é colocado na máquina de ensaios MTS (Material Testing System), conforme descrição a seguir:

Para o teste de propagação estável de trinca, o conjunto da Fig. 14 foi montado numa máquina de ensaios mecânicos servo-hidráulica MTS série 810 , com célula de carga de 50 kN, com características de "closed loop", controle TestStar IIs. Os testes foram controlados pelo deslocamento do atuador, com velocidade de $20 \mu \mathrm{m} \cdot \mathrm{min}^{-1}$. Essas condições foram fixadas a partir de testes preliminares.

Com os dados de deslocamento do atuador e da força instantâneos foram elaboradas curvas carga-deslocamento. A partir dessas curvas foram determinados os trabalhos de fratura, extraindo-se a área sob as mesmas e dividindo-as por duas vezes a área de fratura projetada, de acordo com a equação (C), encontrando-se assim, $\gamma_{\text {wof }}$. A integral sob a curva foi feita até o ponto em que a carga decresce até o valor de $10 \%$ da máxima força atingida durante o ensaio, $\mathrm{P}_{\text {máx }}$. Para cada condição de amostra (composição), foram determinados, no mínimo, 5 valores de $\gamma_{\text {wof }}$ em amostras distintas.

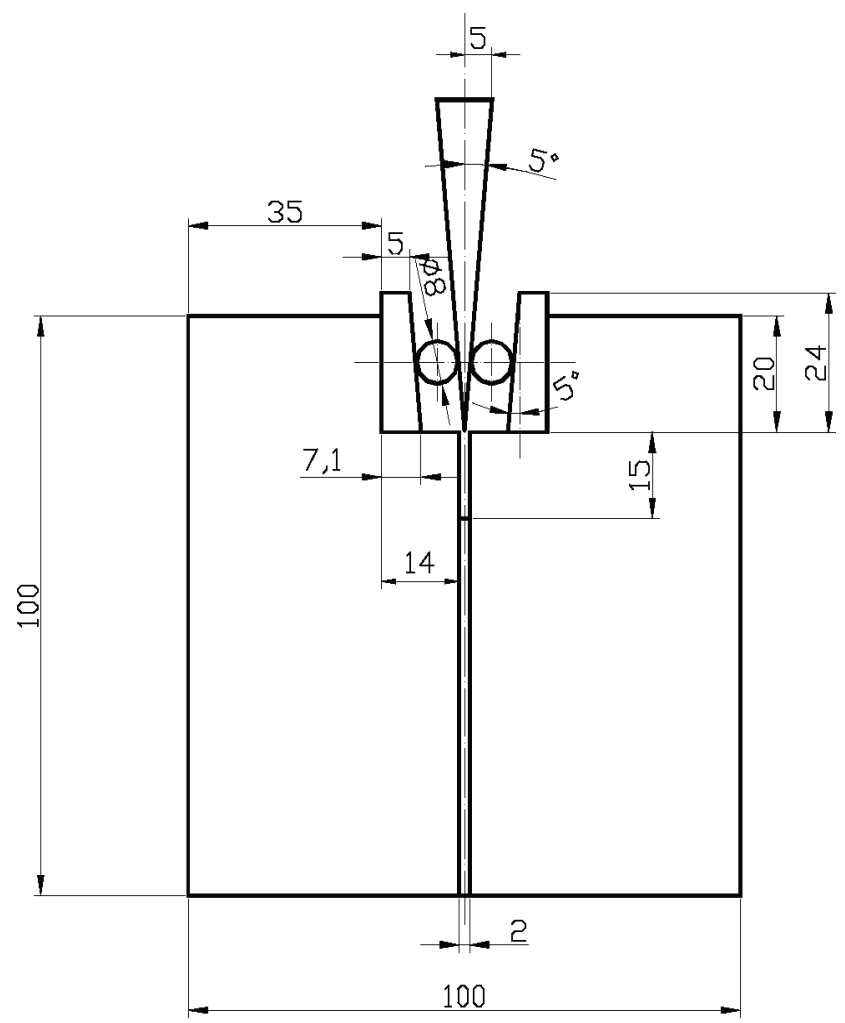

Figura 14: Desenho do conjunto completo para o método da cunha, constituído pelos encostos, roletes, cunha e amostra (dimensões em mm).

[Figure 14: Drawing of the complete set of the wedge splitting method, constituting of supports, rolls, wedge and sample (dimensions in $\mathrm{mm}$ ).] 


\section{RESULTADOS}

A Fig. 15a apresenta uma amostra pronta para montagem do dispositivo da cunha e subseqüente teste de propagação estável de trinca. Nela pode-se notar o perpendicularismo entre a base, o rebaixo, as ranhuras e entalhe, condição necessária para execução do teste. A Fig. 15b ilustra a montagem da amostra com os dispositivos da cunha e os acessórios de acoplamento, cujo conjunto está pronto para ser submetido ao carregamento vertical promovido pelo deslocamento do atuador da máquina de ensaio.

Para avaliar o efeito da composição das argamassas, os resultados de $\gamma_{\text {wof }}$ e de $\mathrm{P}_{\text {máx. }}$, obtidos para as duas composições, estão mostrados na Tabela I. Observa-se que a argamassa com composição 2:1 produziu valores tanto de $\mathrm{P}_{\text {máx }}$ como de $\gamma_{\text {wof }}$ maiores do que aqueles para a composição $3: 1$.

É importante lembrar que se não fosse utilizado o método da cunha, a carga necessária para abertura da trinca

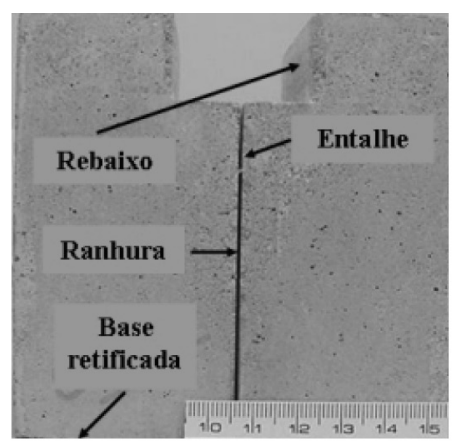

(a)

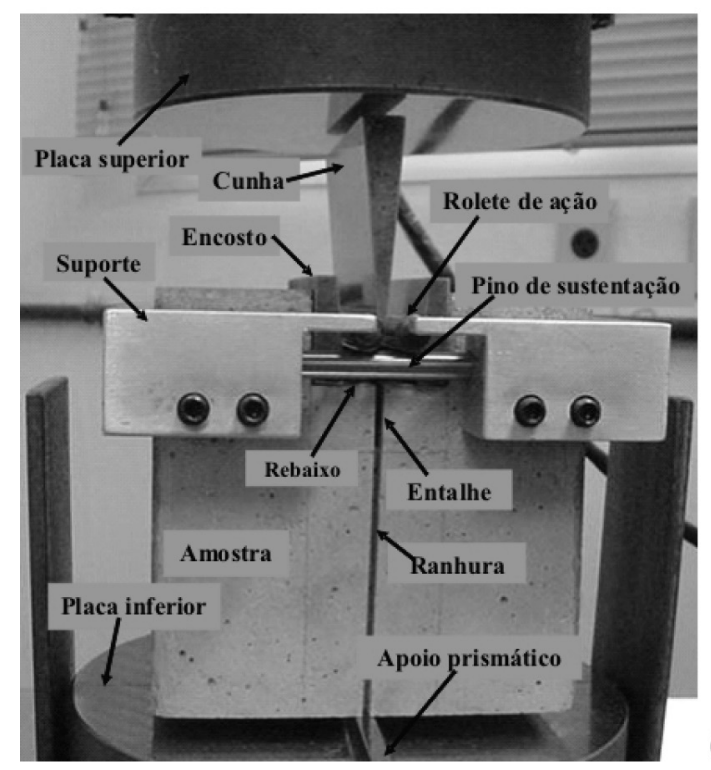

Figura 15: (a) Fotografias de uma amostra moldada com respectivos rebaixo, entalhe e ranhura e (b) Conjunto de amostra e dispositivos, mostrando todos os detalhes de montagem. O sistema está pronto para o início do teste de propagação estável de trinca.

[Figure 15: (a) Photos of the molded sample showing its important parts: rabbet, notch and groove and (b) Arrangement of the sample and devices showing all details of the assembly. The system is ready to start the stable crack propagation test.]
Tabela I - Resultados de energia de fratura e de carga máxima para as argamassas 2:1 e 3:1.

[Table I - Fracture energy and maximum load results for the 2:1 and 3:1 mortars.]

\begin{tabular}{lcclcc}
\hline & \multicolumn{2}{c}{ Composição 2:1 } & & \multicolumn{2}{c}{ Composição 3:1 } \\
\cline { 2 - 3 } \cline { 5 - 6 } Grandezas & $\mathrm{P}_{\text {máx }}(\mathrm{N})$ & $\gamma_{\text {wof }}\left(\mathrm{J} . \mathrm{m}^{-2}\right)$ & & $\mathrm{P}_{\text {máx }}(\mathrm{N})$ & $\gamma_{\text {wof }}\left(\mathrm{J}^{-2} \mathrm{~m}^{-2}\right)$ \\
Média e erro & $377 \pm 69$ & $46 \pm 7$ & & $270 \pm 30$ & $28 \pm 5$ \\
\hline
\end{tabular}

seria da ordem de $2150 \mathrm{MPa}$ e $1540 \mathrm{MPa}$, calculadas pela equação (E) para as composições 2:1 e 3:1, respectivamente, o que poderia levar a uma propagação instável da trinca, já que energia elástica armazenada na máquina seria bem mais elevada, liberando grandes taxas de energia elástica armazenada no sistema, contrariando a condição explicitada pela equação (D).

A Fig. 16 ilustra duas curvas carga-deslocamento típicas obtidas neste trabalho, em que se pode observar um comportamento totalmente estável de propagação de trinca. Observa-se, também, que o trabalho realizado até o ponto de $\mathrm{P}_{\text {máx. }}$ é bem inferior ao trabalho total de fratura, levando a concluir que esse material ainda resiste à propagação de trinca por um período longo mesmo após o início da propagação da trinca. Esse comportamento é mais acentuado quando se observa a Fig. 16a, ou seja, para a argamassa de composição 2:1. Esse material contém relativamente mais cimento, gerando maior coesão tanto na matriz da argamassa como entre a matriz e as partículas maiores da areia. Portanto, maiores forças serão necessárias para a propagação da trinca a partir da ponta do entalhe. Isso consome mais energia por unidade de propagação de trinca e, também, exige maior abertura da boca da trinca para que a sua ponta chegue ao final do corpo de prova, indiretamente indicado pelo maior deslocamento da cunha (atuador). Pela equação (C), podese ver que maiores níveis de força e maiores extensões de deslocamento do ponto de aplicação de carga (topo da cunha) levam a um maior trabalho total realizado pela máquina, para a mesma área de fratura projetada.

Na Fig. 17 apresentam-se fotografias de superfícies de fratura de amostras de argamassas 2:1 (a) e 3:1 (b), e superfície retificada com rebolo diamantado da base da amostra, (c). Pode-se observar o rebaixo, o entalhe, a matriz, os agregados ou partículas de areia e a matriz formada pelo cimento. Também pode ser observada uma distribuição homogênea dos agregados significando que durante a mistura e moldagem com vibração não houve segregação das partículas para a parte inferior da amostra.

Os efeitos de preparação das amostras e da observação de todos os parâmetros operacionais para os testes de propagação estável de trinca pelo método da cunha mostrados neste trabalho vêm contribuir para um melhor estabelecimento dos procedimentos de aplicação desta metodologia, que tem se destacado como fundamental para uma confiável determinação experimental da energia de fratura para materiais cerâmicos de microestrutura grosseira, tais como as argamassas e os concretos refratários. 

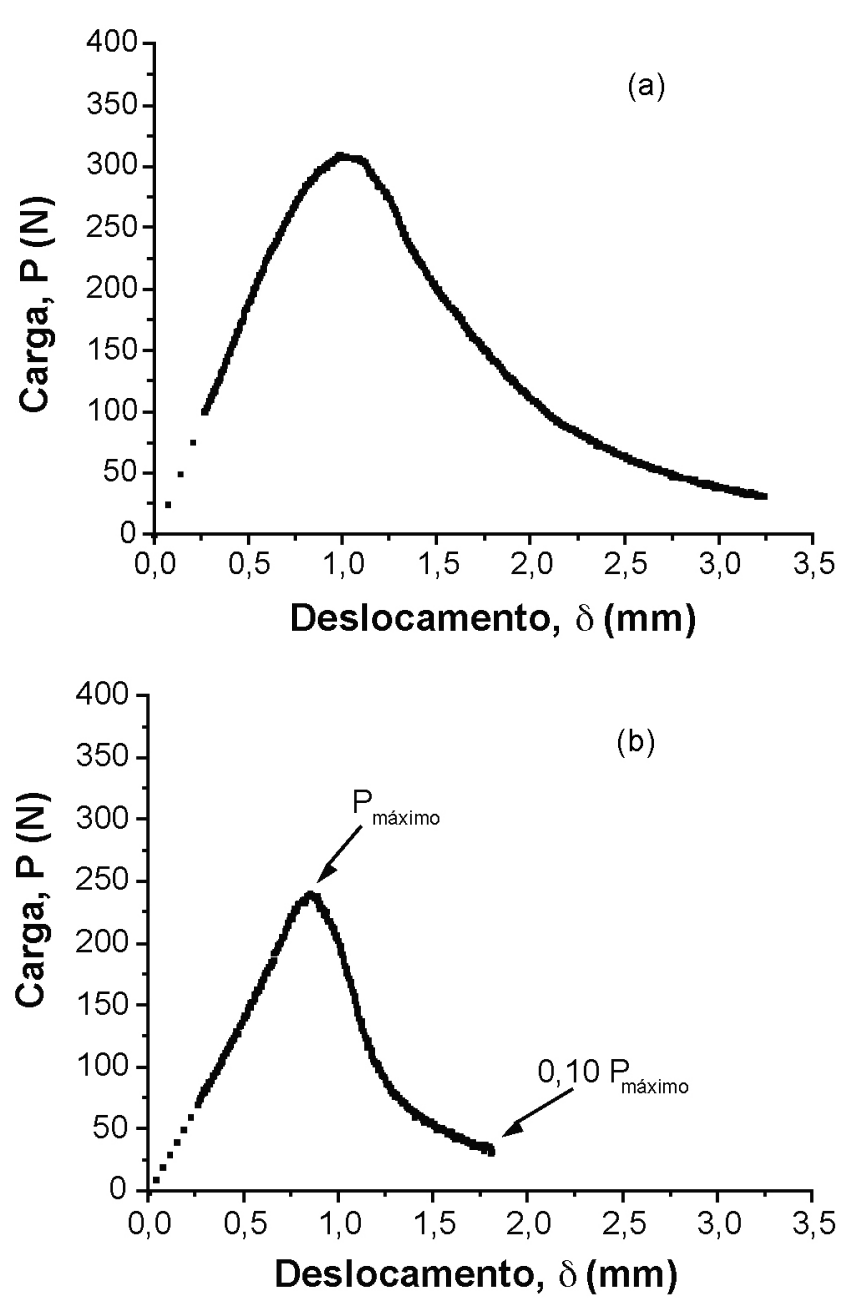

Figura 16: Curvas carga-deslocamento típicas de duas amostras ensaiadas: argamassa (a) 2:1 e (b) 3:1.

[Figure 16: Typical load-displacement curves of two tested samples: (a) 2:1 mortar and (b) 3:1 mortar.]

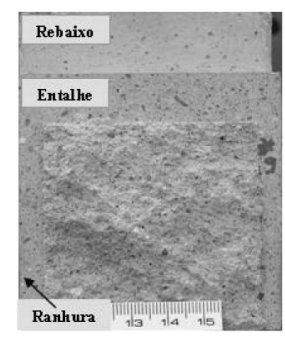

(a)

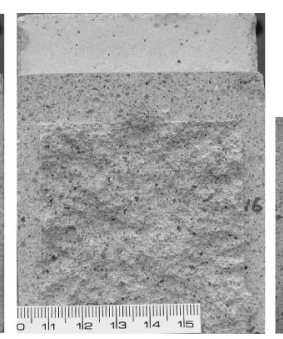

(b)

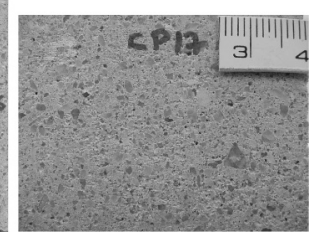

(c)
Figura 17: Fotografias das amostras das argamassas (a) 2:1 e (b) 3:1. Nelas podem ser visto a superfície do rebaixo com acabamento produzido na moldagem, à superfície do entalhe e ranhuras produzidos com disco diamantado e superfície de fratura. Em (c) observa-se detalhes da superfície retificada com rebolo diamantado da base do corpo de prova.

[Figure 17: Photos of the mortar samples (a) 2:1 and (b) 3:1. In them can be seeing the rabbet surface with surface quality of the $P V C$ mold, notch and grooves surfaces produced by diamond disc and fracture surface. In (c) it is shown details of the bottom surface of the sample finished with a diamond wheel.]

\section{CONCLUSÕES}

O método da cunha é perfeitamente aplicável para amostras com tamanhos relativamente grandes, o que favorece a geração de resultados mais precisos, confiáveis e menos dispersos para materiais com microestruturas grosseiras, como no caso de argamassas e concretos de modo geral.

No projeto e na construção das peças que constituem os dispositivos e na montagem do sistema, a precisão e o alinhamento é de absoluta necessidade, pois quaisquer erros levam a resultados insatisfatórios.

Moldes de PVC são perfeitamente viáveis para produção das amostras para o teste de propagação estável de trincas de argamassas e concretos, pois produzem superfícies com acabamento final de uso, não necessitando de usinagem das partes que ficam em contato com as paredes do mesmo.

O material selecionado para a construção do dispositivo da cunha e do acoplamento à máquina de ensaio, aço VC131, foi perfeitamente adequado, porém um tratamento térmico de têmpera e o polimento das faces de contato são necessários para todas as peças envolvidas.

O método da cunha mostrou-se sensível à força máxima e à energia de fratura das duas argamassas empregadas neste trabalho, mostrando direta correlação entre esses parâmetros e a coesão do material.

A metodologia completa de preparação e ensaio dos corpos de prova pelo método da cunha para argamassas e concretos de um modo geral, encontra-se disponível no laboratório do GEMM/DEMa/UFSCar, que foi o primeiro laboratório a adaptar e adotar esse método no Brasil e, em breve, o estará também na Escola de Engenharia de Lorena.

\section{AGRADECIMENTOS}

À FAPESP (Proc. 2001/04324-8), ao CNPq pelas bolsas de Produtividade em Pesquisa (Procs. 304980/2003-0 e 301073/2006-6), pelo projeto Universal (Proc. 470504/20048) e pela bolsa Pós-Doutorado Sênior (Proc. 150038/20062). À aluna de graduação Giseli C. R. Garcia, EEL-USP, pela contribuição na correção e discussão do trabalho. Ao técnico Carlos R. Bernardo, EEL-USP, pela correção dos desenhos envolvidos no projeto e ao Sr. Ricardo V. C. Martins pelo auxílio na obtenção das fotografias das amostras.

\section{REFERÊNCIAS}

[1] E. Tschegg, Prüfeinrichtung zur Ermittlung von bruchmechnishen Kemwerten sowie hierfür geeignete, Prüfkörper, Austrian Pat. AT 390328B, registered (1986).

[2] W. E. Lee, W. Vieira, S. Zhang, K. G. Ahari, H. Sarpoolaky, C. Parr, Int. Mater. Rev. 46, 3 (2001) 145.

[3] F. N. Cunha-Duncan, R. C. Bradt, Cerâmica 49 (2003) 199.

[4] Y-C. Ko, Ceram. Int. 27 (2001) 501

[5] B. Cotterell, S. W. Ong, C. Qin, J. Am. Ceram. Soc. 78 (1995) 2056. 
[6] D. P. H. Hasselman, J. Am. Ceram. Soc. 46 (1963) 535.

[7] D. P. H. Hasselman, J. Am. Ceram. Soc. 52 (1969) 600.

[8] C. Aksel, P. D. Warren, F. L. Riley, J. Eur. Ceram. Soc. 24 (2004) 2407.

[9] D. R. Larson, J. A. Coppola, D. P. H. Hasselman, J. Am. Ceram. Soc. 57 (1974) 417.

[10] B. Alapin, M. Ollig, J. Pötschke, in Proc. 46 ${ }^{\text {th }}$ Int. Coll. Refr. Eurogress, Aachen, Alemanha (2003) 107.

[11] H. Harmuth, K. Rieder, M. Krobath, E. Tschegg, Mater. Sci. Eng. A 214 (1996) 53.

[12] O. Peitl, E. D. Zanotto, J. Non-Cryst. Sol. 247 (1999) 39.

[13] C-C. Chou, I-L. Cheng, K-J. Chen, Y-C. Ko, Am. Ceram. Soc. Bull. 65 (1986)1042.

[14] R. Nilica, H. Harmuth, Cem. Conc. Res. 35 (2005)
1641.

[15] E. K. Tschegg, M. Elser, S. E. Stanzl-Tscgegg, Cem. Concr. Comp. 17 (1995)57.

[16] H. Harmuth, Theor. Appl. Frac. Mec. 23 (1995)103.

[17] C. Rosselló, M. Elices, G. V. Guinea, Cem. Conc. Res. 36 (2006) 1345.

[18] J. Nakayama, H. Abe, R. C. Bradt, J. Eur. Ceram. Soc. 64 (1981) 671.

[19] X. Hu, K. Duan, Cem. Conc. Res. 34 (2004)1321.

[20] R. Walter, L. Ostergaard, J. F. Olesen, H. Stang, Eng. Fract. Mech. 72 (2005) 2565.

[21] B. L. Karihaloo, H. M. Abdalla, Q.Z. Xiao, Cem. Conc. Res. 36 (2006) 171.

(Rec. 04/12/2007, Ac. 08/02/2008) 